\title{
Local Magnetic Properties in Non-oriented Electrical Steel and Their Dependence on Magnetic Easy Axis and Misorientation Parameters
}

\author{
MATTHEW GALLAUGHER, ARASH SAMIMI, THOMAS W. KRAUSE, \\ LYNANN C. CLAPHAM, and RICHARD R. CHROMIK
}

\begin{abstract}
An understanding of how material parameters, especially orientation and misorientation, influence the magnetic properties of non-oriented electrical steel (NOES) is important for improving the efficiency of the material in service. In this study, the local magnetic properties were measured using magnetic Barkhausen noise $(\mathrm{MBN})$ on different test locations on different strips of NOES material. Local variations in magnetic properties, texture, and misorientation were revealed. A new interpretation for misorientation, called the easy axis misorientation (EAM), was created to describe the alignment of the magnetic easy axes between neighboring grains. This new EAM, visualized as a single value parameter or graphed as a distribution, was shown to be more effective at predicting the isotropic magnetic properties than previously used texture parameters based on standard orientation/misorientation definitions. It was found that a larger EAM value, especially when associated with a lower small angle EAM intensity distribution, was associated with a larger MBN energy. A larger MBN energy has been previously associated with lower losses, and therefore a greater material efficiency.
\end{abstract}

DOI: $10.1007 / \mathrm{s} 11661-014-2728-9$

(C) The Author(s) 2014. This article is published with open access at Springerlink.com

\section{INTRODUCTION}

ELECTRICAL steels, with the major alloying elements of silicon ( $\sim 1$ to $4 \mathrm{wt}$ pct $)$ and aluminum $(\sim 0.5 \mathrm{wt} \mathrm{pct})$, are important materials for magnetic flux carrying applications. Electrical steels can come in different forms which are tailored to their applications, mostly through the variations in texture. ${ }^{[1,2]}$ The variations in magnetic properties due to texture are a result of magnetocrystalline energy, which for electrical steels determine that it takes the least energy to magnetize the material along the $\langle 001\rangle$ family of directions (the easy axis direction), and the most energy to magnetize the material along the $\langle 111\rangle$ family of directions (the hard axis direction). ${ }^{[3]}$

Non-oriented electrical steels (NOES) are designed to have optimum magnetic properties under rotating magnetic fields. As a result, from a texture perspective, it is desirable for NOES to have a maximum of easy axis directions in the plane of the sheet (the rolling directiontransverse direction plane, or RD-TD plane), but without a preferred orientation such that the magnetic

MATTHEW GALLAUGHER, Ph.D. Candidate, and RICHARD R. CHROMIK, Associate Professor, are with the Department of Mining and Materials Engineering, McGill University, 3610 University Street, Montreal, QC H3A 0C5, Canada. Contact e-mail: richard.chromik@mcgill.ca ARASH SAMIMI, Ph.D. Candidate, and LYNANN C. CLAPHAM, Professor, are with the Applied Magnetics Group, Queen's University, Kingston, ON K7L 3N6, Canada. THOMAS W. KRAUSE, Professor, is with the Department of Physics, Royal Military College of Canada, Kingston, ON K7K 7B4, Canada.

Manuscript submitted July 31, 2014.

Article published online January 7, 2015 properties are the same in all directions (i.e., isotropic magnetic properties). The measurement and quantification of texture quality can be complicated for NOES since any possible crystal orientation may be present. This has spawned several different texture parameters that have been used to link the texture to the magnetic properties.

The simplest texture parameter is texture factor (TF), which is the volume fraction of planes within the cube fiber $\{100\}\langle u v w\rangle$ divided by the volume fraction of planes within the gamma fiber $\{111\}\langle u v w\rangle .{ }^{[4,5]}$ Since two easy axis directions lie in each $\{100\}$ plane, a higher value of TF is associated with better magnetic properties, namely lower loss and higher permeability. ${ }^{[4,5]}$ This texture parameter is non-directional, in that its value is not associated with a magnetization direction. There are two main weaknesses in TF: (1) only the orientations present with planes close to $\{100\}$ and $\{111\}$ orientations are considered in the calculation, and (2) the contribution from the easy axes present in $\{h k 0\}$ planes is ignored.

Correspondingly, past research has introduced different texture parameters to deal with these issues by studying the orientation of the easy axis directions themselves, rather than the planes that they are associated with. These have included the magnetic texture factor (MTF), ${ }^{[6]}$ the A parameter, ${ }^{[7-12]}$ and texture calculations based on the formula for magnetocrystalline anisotropy. ${ }^{[13-16]}$ However, these texture parameters are typically directional, in that they are calculated with respect to a magnetization direction of interest, typically the RD. This can allow for the better prediction of measured magnetic properties, since the magnetic prop- 
erties of NOES are typically measured in one direction using Epstein frame or single strip testing. Although they can predict measured magnetic properties well, for NOES that will be exposed to rotating magnetic fields, it is not clear whether these directional-based magnetic and texture comparisons are a good representation of the material's performance in the field.

In addition to the directionality associated with the magnetic testing and materials characterization of NOES, these tests are typically performed over a bulk length scale where it is difficult to separate the influence of the misorientation from the overall orientation. Magnetic Barkhausen noise (MBN) testing is a technique that measures the induced voltage changes from the abrupt Barkhausen jumps during a magnetization cycle, and can be linked to microstructural properties at a more local scale. For electrical steels, MBN testing has typically been applied to grain-oriented electrical steels (GOES), which have a simpler microstructure than NOES, characterized by a larger grain size and a strong Goss $(\{110\}\langle 001\rangle)$ texture. Yamaura et al. found that Barkhausen Noise power (analogous to MBN energy) increased with increasing grain boundary misorientation angle, however, the study was mostly restricted to low angle grain boundaries and did not include high angle or coincident site lattice (CSL) boundaries. ${ }^{[17]}$ It should also be noted that due to the nature of GOES, it could not be determined whether the increase in MBN power was from the grain boundary character itself, or from the difference in the easy axis directions between the grains. ${ }^{[17]}$

In similar work involving misorientation, Kawahara et al. showed that the GOES domain structure was continuous across low angle grain boundaries, but a high angle grain boundary caused a discontinuous domain structure or a continuous but inclined domain structure. ${ }^{[18]}$ The difference between the two high angle grain boundary domain structures was attributed to the alignment of the easy axes, as opposed to the misorientation. ${ }^{[18]}$ The criteria for continuity of the domain structure across the high angle GB were related to the orientation between each of the easy axes and the grain boundary normal, ${ }^{[18]}$ which determines the magnetostatic energy across the GB. ${ }^{[19]}$ This showed the difference between grain boundary character, or pure misorientation, and the deviation of the easy axis. Also, when an external magnetic field was applied, the domain structure surrounding a low angle grain boundary changed immediately in a significant manner, while the domain structure surrounding a high angle GB, under the same conditions, changed in a smooth, non-abrupt manner. ${ }^{[18]}$ This implied that the interaction between domain walls and low angle GB was weaker than that of the high angle GB.

Similarly, recent work has revealed that magnetic domain continuity in NOES materials can be present when the angles between the easy axes of the two grains are the same with respect to the normal to the grain boundary, ${ }^{[20]}$ but these effects on the overall magnetic properties of NOES are presently unknown. In addition, work by Samimi et al. has shown that for NOES, the $\mathrm{MBN}$ energy correlated inversely with loss. ${ }^{[15]} \mathrm{A}$ similar result was shown by Birsan et al. for GOES. [21] Consequently, it is believed that MBN energy can also be applied to NOES to study the effects of misorientation and orientation, and be correlated to loss properties.

Compared to NOES, the misorientation for GOES is relatively straightforward, since small deviations from the Goss texture can be more easily visualized as deviations of the single easy axis of interest aligned in the RD. In the case of NOES, however, standard misorientation information is not necessarily related to the magnetic easy axis or magnetic properties. Although misorientation can be represented in different ways, the most common method is a common axis of rotation, and an angle of rotation describing how to transform one crystal to the other crystal's orientation. ${ }^{[22]}$ Due to cubic symmetry, for NOES, there are 24 ways to describe a misorientation, so there are 24 possible axes to describe it. ${ }^{[2]}$ Typically, the solution with smallest angle of rotation, termed the disorientation, is chosen. ${ }^{[22]}$ From a magnetic point of view, however, these possible misorientation axes are not equivalent due to magnetocrystalline anisotropy. As a result, for NOES, where any misorientation relationship is possible, it is difficult to relate the disorientation to the magnetic easy axis positions in order to investigate the impact of misorientation on magnetic properties. Consequently, misorientation has not been typically studied in NOES in terms of its impact on magnetic properties.

In order to more accurately predict the efficiency of NOES under rotating magnetic fields, it would be necessary to use a non-directional texture parameter, as well as a way to quantify the influence of neighboring grains in the form of a misorientation parameter. Recent work has shown that the minimum angle between an easy axis and the RD-TD plane, called the $\beta$ parameter, was linked to the magnetic domain structure and alignment of NOES. ${ }^{[20]}$ Since $\beta$ is also a non-directional texture parameter, it may be ideal to predict the material's isotropic magnetic properties. In addition, as previously mentioned, misorientation could be studied by using the local area testing capabilities of MBN. MBN could be applied to study both texture issues, in that it could be measured in multiple directions in the same area and averaged, revealing isotropic magnetic properties that are more representative of the material's properties under rotating applied magnetic fields.

The purpose of this work was to characterize the local isotropic magnetic properties of NOES and match these properties to orientation and misorientation properties. The testing was conducted on multiple locations on strip samples from a single manufacturer in the semi-processed and fully processed conditions to eliminate the influence of sample thickness, grain size, and chemistry. The accuracy of the texture parameter $\beta$ in predicting these isotropic properties was tested, and compared to the standard TF. Moreover, the influence of misorientation on the magnetic properties was explored with standard misorientation as well as a new misorientation parameter, the easy axis misorientation (EAM). This EAM is a newly created misorientation parameter that describes the degree of EAM between grains. If texture 
parameters involving orientation and misorientation can be used to accurately predict isotropic magnetic properties, it is believed that this will be a more accurate way to predict the material's performance in rotating magnetic field applications, such as the drivetrains of electric car motors.

\section{MATERIALS AND METHODS}

Strips of semi-processed (Sample A) and fully processed (Sample B) NOES from the same manufacturer with compositions shown in Table I were selected. The difference between the two samples types is that fully processed samples are subjected to a final stress relief annealing treatment, while semi-processed samples are not. From each sample, five locations were chosen for magnetic testing measurement by MBN. Sampling different locations on the same strips was done to minimize the influence of grain size, chemistry, and sample thickness on the magnetic properties.

The magnetic measurements at the five locations were performed using a flux-controlled Barkhausen noise system at Queen's University. The MBN apparatus consisted of a surface dual-core probe (tetrapole) ${ }^{[23,24]}$ in combination with an in-house designed magnetic flux controller which controlled the flux wave-form to obtain consistency and repeatability in local MBN measurements. ${ }^{[23,25]}$ More details about the testing method and apparatus can be found elsewhere. ${ }^{[15]}$

In simplified terms, the MBN measurement involved the application of an alternating magnetic field in the plane of the sample, and measuring the induced voltage caused by the vertical magnetization components projected out of the sample's surface. The rms envelope of the Barkhausen signal is measured, and the MBN energy, defined as the time integral of the voltage squared signal, is evaluated. In this case, flux-controlled MBN measurements were taken at a $30 \mathrm{~Hz}$ excitation frequency and $100 \mathrm{mT}$ flux density measured at the excitation magnet poles, which is approximately equivalent to $1 \mathrm{~T}$ within the samples, accounting for sample thickness. The sensing radius of the instrument at $30 \mathrm{~Hz}$ has been estimated to be approximately $2.4 \mathrm{~mm} .{ }^{[24]}$ To provide isotropic magnetic properties that may be a better representation of the material's magnetic properties under rotating fields, directional MBN measurements were performed in 12 directions between the RD and the TD of the sample and averaged (referred to as the average $\mathrm{MBN}$ energy).

After the MBN testing, the sampling locations were cut out, ground, and polished with $3 \mu \mathrm{m}$ and then $1 \mu \mathrm{m}$ oil-based diamond solution. For the final polish, a vibratory polisher and $0.05 \mu \mathrm{m}$ colloidal silica were used for approximately 8 hours. After polishing, a low magnification electron backscatter diffraction (EBSD) map was collected using a Philips XL-30 field emission scanning electron microscope (FE-SEM) with a TSLEBSD system using the orientation imaging microscopy (OIM) TSL software. Using offline TSL Analysis software, the maps were processed, and used to produce inverse pole figure (IPF) maps as well as orientation distribution functions (ODFs) to describe the texture. From this, the TF was calculated using a tolerance of 15 deg. The TSL analysis software was also used to calculate a correlated misorientation distribution function (MDF) and the grain boundary character distribution (GBCD) to describe the misorientation of each testing location. By using a correlated MDF, the nearest neighbor information was retained, in that the misorientation is only measured between a grain and its nearest neighbors.

The ODF and MDF from the TSL software were both exported and used to calculate the easy axis-related texture relationships. To calculate the $\beta$ parameter, which is the angle between the nearest easy axis and the RD-TD plane of the sample, the ODF was exported to MATLAB and then the results were graphed using Origin software, as outlined in Reference 20. To calculate the EAM parameter, the MDF data was exported, and the calculation was done as outlined in the following section. The EAM was also calculated as a distribution, and plotted as a 3D colored scatter plot, such that the $X, Y$, and $Z$ axes represent the misorientation from the three easy axes, and the color indicated the normalized weighting of the misorientation from the MDF.

\section{A. Calculation of Easy Axis Misorientation (EAM) Parameter and Distribution}

In its simplest form, the definition for a crystal's orientation relative to a reference orientation is given by the matrix $g,{ }^{[22]}$

$$
g=\left(\begin{array}{ccc}
\cos a_{1} & \cos b_{1} & \cos c_{1} \\
\cos a_{2} & \cos b_{2} & \cos c_{2} \\
\cos a_{3} & \cos b_{3} & \cos c_{3}
\end{array}\right)
$$

The $g$ matrix is a $3 \times 3$ rotation matrix of direction cosines that define the transformation between the crystal reference system $r_{\mathrm{c}}$ (defined with axes aligned in the [100], [010], and [001] directions) and the crystal orientation $r_{\mathrm{s}}$ of the sample coordinate system by ${ }^{[22]}$

$$
r_{\mathrm{c}}=g \cdot r_{\mathrm{s}}
$$

For the components in the $g$ matrix, the subscript indicates the axis of the sample (1: $X$ axis, $2: Y$ axis, and

Table I. Chemical Composition of Major Alloying Elements of the NOES Samples Examined (Weight Percent, Measured by Inductively Coupled Plasma Atomic Emission Spectroscopy (ICP-AES) and Combustion Methods)

\begin{tabular}{lccccccccc}
\hline Sample & $\mathrm{C}$ & $\mathrm{Al}$ & $\mathrm{Mn}$ & $\mathrm{P}$ & $\mathrm{Si}$ & $\mathrm{N}$ & $\mathrm{O}$ & $\mathrm{Fe}$ & Sample Thickness (mm) \\
\hline A & 0.007 & 0.54 & 0.21 & 0.022 & 3.27 & 0.002 & 0.007 & balance & 0.350 \\
B & 0.006 & 0.53 & 0.20 & 0.020 & 3.26 & 0.002 & 0.006 & balance & 0.350 \\
\hline
\end{tabular}


3: $Z$ axis), and the letter indicates the axis of the crystal reference system (a: $X$ axis, b: $Y$ axis, and c: $Z$ axis). For example, $\cos a_{2}$ is the angle between the $Y$ axis of the sample and the $X$ axis of the reference system, as shown in Figure 1.

This $g$ matrix can also be used to describe misorientation, or in other words, the transformation between the reference system defined by the orientation of one grain (grain 1) rather than a sample reference system, relative to the orientation of a second grain (grain 2). In this case, the misorientation matrix $M_{12}$ can be defined from the $g$ matrices of the two crystal orientations $\left(g_{1}\right.$ and $\left.g_{2}\right)$ with the following equation ${ }^{[22]}$ :

$$
M_{12}=g_{1}^{-1} g_{2}
$$

If we consider the $M_{12}$ matrix, the $i$ th column will represent the three angles between the three reference axes of grain 2 and the $i$ th reference axis of grain 1. For NOES, these axes are the magnetic easy axes. In other words, the minimum angle given in the ith column of the $M_{12}$ matrix will reveal the minimum easy axis deviation for the $i$ th axis between the two grains. This M matrix was calculated for each orientation from the exported correlated MDF data using code written in MATLAB by converting the Euler angles in the MDF to the $M$ matrix using equations presented elsewhere. ${ }^{[22]}$

For the whole matrix, the three angles representing the EAM can then be chosen from the $M$ matrix values as the minimum value for each column. The average of these three angles, multiplied by the normalized weight from the correlated MDF, summed up over all the misorientations yields the EAM parameter. The EAM distribution is graphed by plotting a $3 \mathrm{~d}$ scatter plot with the three angles as the three axes in the plot, and a color value is assigned based on the weight from the MDF.

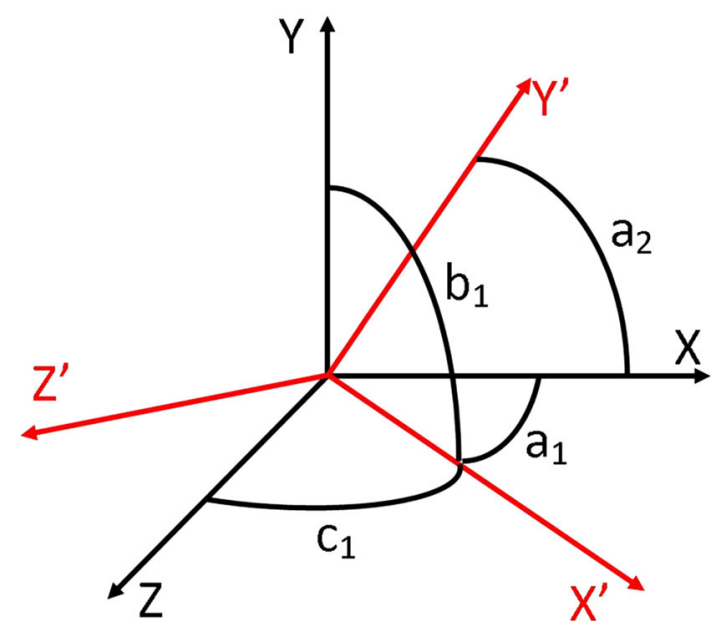

Fig. 1-Schematic showing the relation between the sample coordinate system (red) from Eq. [2] (or grain 1, $g_{1}$ in Eq. [3]) and the crystal reference coordinate system (black) from Eq. [2] (or grain 2, $g_{2}$ in Eq. [3]) as described by the angles defined by the $g$ matrix in Eq. [1] (Color figure online).

\section{RESULTS}

A. General Texture: Orientation Distribution Functions (ODFs)

The $\varphi_{2}=45$ deg section of the ODFs for the semiprocessed (A) and fully processed (B) sample test locations is presented in Figures 2 and 3, respectively. Although the texture present in the different locations was generally similar, variations were observed. The ODF information was taken from the entire EBSD map, which encompassed approximately 250 grains, with an average grain diameter of approximately $140 \mu \mathrm{m}$.

\section{Semi-processed}

For sample A, the major texture components in most cases were the cube fiber $\{100\}\langle u v w\rangle$ and the gamma fiber $\{111\}\langle u v w\rangle$. Location A2 appeared to have the strongest cube fiber, and both locations A1 and A2 appeared to have the strongest gamma fiber texture components. Each location also showed a texture component running from the cube fiber to the gamma fiber at a $\varphi_{1}$ of approximately $25 \mathrm{deg}$, referred to as the $\alpha^{*}$ component. Based on the ODFs, it is expected that the A2 test location would exhibit the highest MBN energy (indicating lower losses according to ${ }^{[15,21]}$ ), since it possessed more easy axes present in the plane of the sample, as indicated by the higher cube fiber component.

\section{Fully processed}

The fully processed samples (B) had relatively consistent texture components with some minor variations. The main components were again the cube and gamma fiber, as well as the $\alpha^{*}$ texture component running from the cube fiber to the gamma fiber at a $\varphi_{1}$ of approximately $25 \mathrm{deg}$. All of the samples had relatively strong cube fiber components except for B2, and samples B2, $\mathrm{B} 3$, and B5 had the highest gamma fiber component. It is expected that locations $\mathrm{B} 1, \mathrm{~B} 3$, and $\mathrm{B} 4$ would have the highest MBN energy since they appeared to have a high cube fiber component while also possessing a less significant gamma fiber component.

When comparing samples A to $\mathrm{B}$, the fully processed B samples appeared to have a less significant gamma fiber component. From this, it is expected that the samples from B would have a higher MBN energy than the samples from A, without considering any other of the variables influencing magnetic properties.

\section{B. Inverse Pole Figure and $\beta$ Parameter Maps}

\section{Semi-processed}

The texture distribution and general grain structure of the semi-processed sample (sample A) were revealed by the IPF map (Figure 4(a)) and the $\beta$ parameter map (Figure 4(b)). As discussed previously, ${ }^{[20]}$ the $\beta$ parameter is a more representative way to display the orientation of the easy axis to the RD-TD plane of the sample, since it displays the angle between the closest easy axis and the RD-TD surface for all orientations. Each test location showed a similar grain size, with some variations in texture distribution, consistent with the observations in the ODF's. 


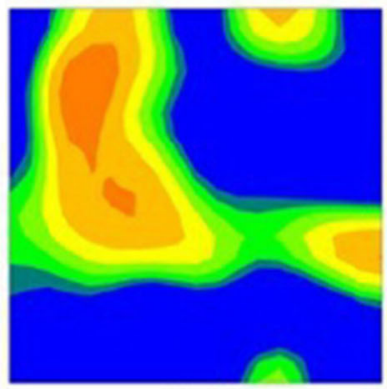

$45^{\circ}$

(a)

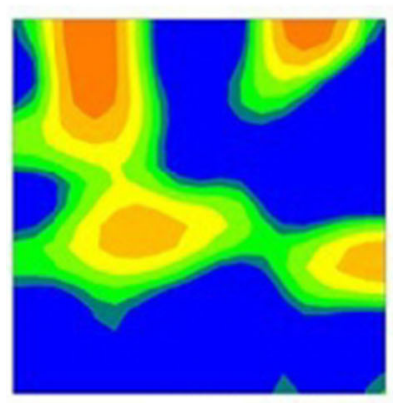

$45^{\circ}$

(c)

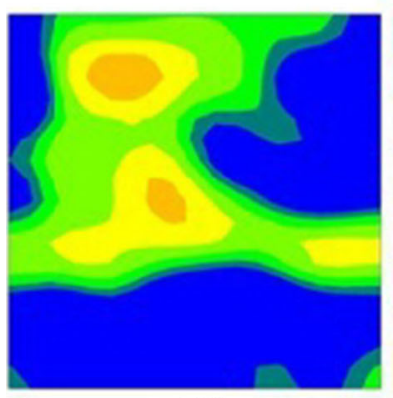

$45^{\circ}$
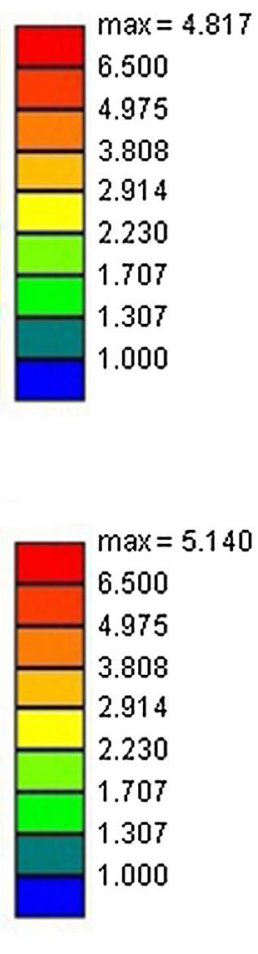

$\max =5.140$
6.500
4.975
3.808
2.914
2.230
1.707
1.307
1.000

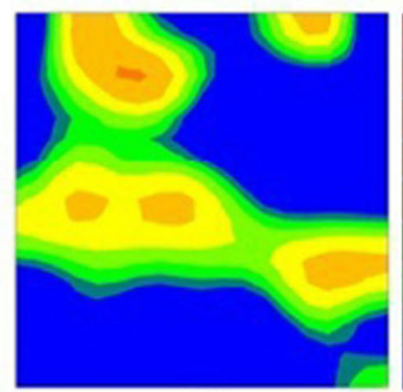

$45^{\circ}$

(d)

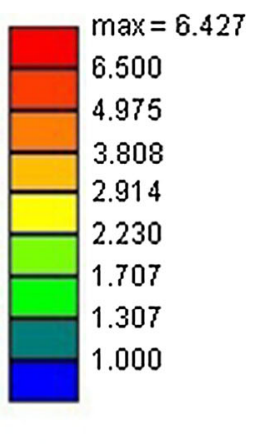

(b)

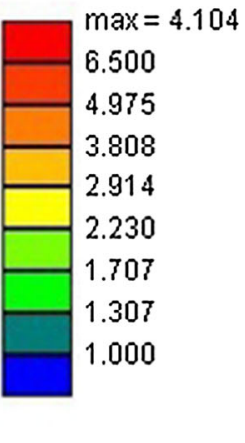

$\max =4.104$

.500

4.975

2914

2.230

1.707

1.307

1.000

\section{(e)}

Fig. 2 - Orientation distribution function (ODF) sections for the $\varphi_{2}=45$ deg sections for samples A1 (a) through A5 (e), where the $x$ axis is $\varphi_{1}$, and the $y$ axis is $\Phi$, both increasing from the top left corner of the ODF sections. All samples had varying cube fiber and $(\Phi=0$ deg, along the top edge) gamma fiber components $(\Phi=55 \mathrm{deg}$, horizontal line in the lower half), along with a component joining the cube and gamma components at roughly $\varphi_{1}=25 \mathrm{deg}$.

\section{Fully processed}

The texture distribution and general grain structure of the fully processed sample (sample B) were also revealed by the IPF map (Figure 5(a)) and the $\beta$ parameter map (Figure 5(b)). The grain size of the fully processed samples was similar in all test locations, and was also similar to the grain size in the semi-processed sample. However, there were small variations in texture between the test locations of the fully processed samples, again consistent with the ODF observations. In addition, it appeared that the $\beta$ parameter values for the fully processed test locations were smaller (indicating a more favorable texture) than the semi-processed test locations, visualized by more grains that were blue/cyan rather than orange/red when comparing Figures 5(b) to 4(b).

\section{Magnetic Properties vs Texture Parameters and Orientation}

The average MBN energy values were directly compared to the overall grain diameter, texture factor (TF), and $\beta$ parameter values for each sample location. The grain diameter between all the different regions was essentially the same $(\sim 140 \mu \mathrm{m})$, so no significant influence of grain size on the MBN energy was present. The fully processed samples in general appeared to have a higher texture factor and a lower $\beta$ parameter value 


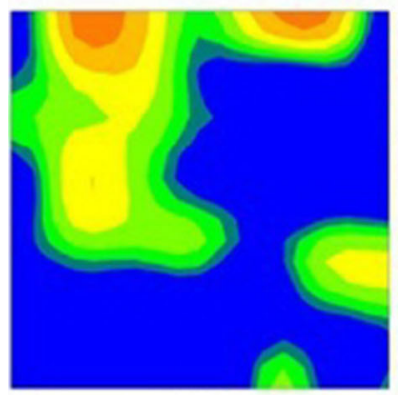

$45^{\circ}$

(a)

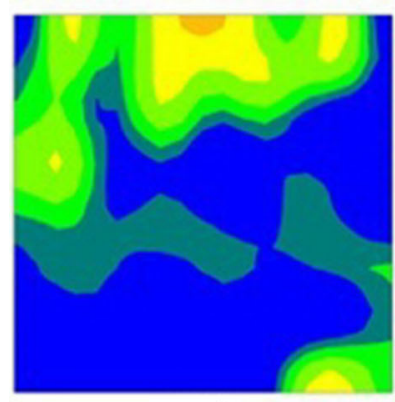

$45^{\circ}$

(c)

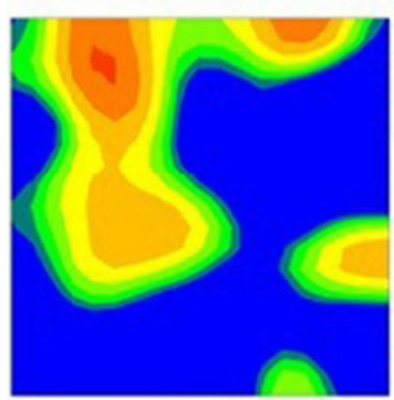

$45^{\circ}$

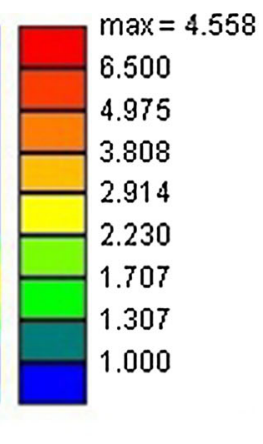

$\max =4.102$

6.500

4.975

3.808

2.914

2.230

1.707

1.307

1.000
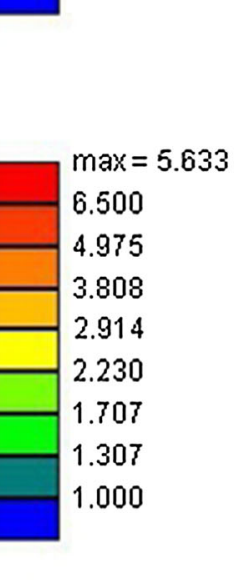
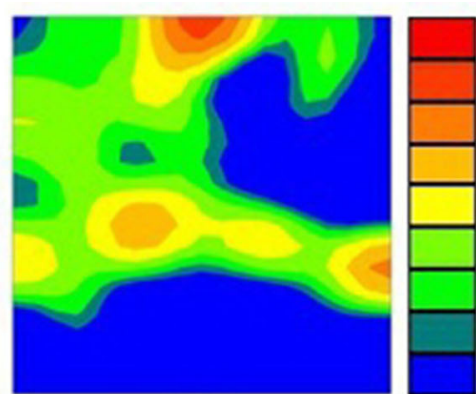

$\max =5.933$

6.500

4.975

3.808

2.914

2.230

1.707

1.307

1.000

$45^{\circ}$

(b)
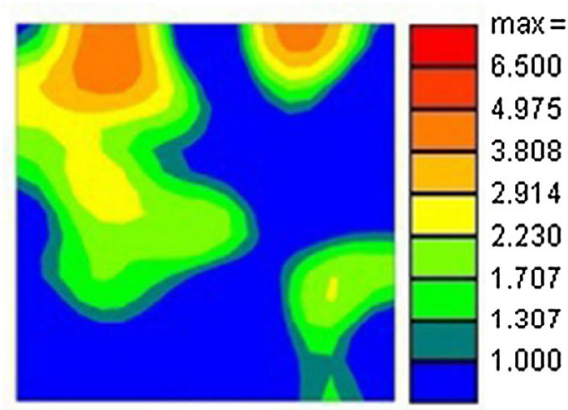

$45^{\circ}$

(d)

(e)

Fig. 3-Orientation distribution function (ODF) sections for the $\varphi_{2}=45$ deg sections for samples B1 (a) through B5 (e), where the $x$ axis is $\varphi_{1}$, and the $y$ axis is $\Phi$, both increasing from the top left corner of the ODF sections. All samples had varying cube fiber and ( $\Phi=0$ deg, along the top edge) gamma fiber components $(\Phi=55 \mathrm{deg}$, horizontal line in the lower half), along with a component joining the cube and gamma components at roughly $\varphi_{1}=25 \mathrm{deg}$.

(indicating a better texture) than the semi-processed samples, consistent with the observations in the $\beta$ parameter maps (see Figure 6).

The variation in MBN energy between different locations on the two strips was significant, only slightly less than the change in values observed between different manufacturers. ${ }^{[15]}$ Similarly, the TF variations for the test locations also showed variations on the order of variations observed between samples of different manufacturers. ${ }^{[6]}$ This demonstrated that local variations in microstructural parameters and magnetic properties were present in the NOES strips. For easier comparison, the average MBN energy was plotted vs TF (Figure 6(a)) and $\beta$ (Figure 6(b)) for each test location on both the semi-processed and fully processed samples. For these graphs and the following graphs, linear trend lines were arbitrarily chosen as a way to compare the relationships between the orientation/misorientation parameter and the average MBN energy.

\section{Semi-processed}

From the plots of average MBN energy (from 12 directions) vs $\mathrm{TF}$ and $\beta$ (black squares in Figures 6(a) and (b)), no significant trends for the semi-processed sample (A) were observed. In both cases, the main reason for the poor fit for the semi-processed samples was A4, which showed a high average MBN energy, despite a low $\mathrm{TF}$ and a high $\beta$ parameter. 

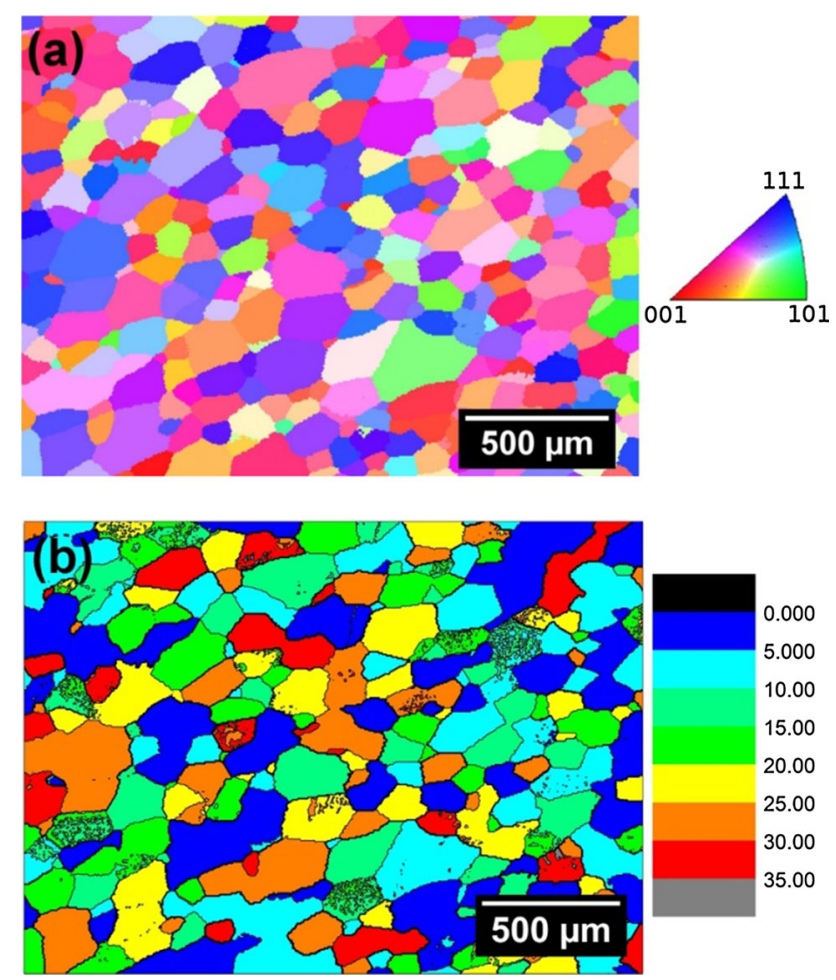

Fig. 4-Inverse pole figure (IPF) map in the ND $(a)$ and the $\beta$ parameter map $(b)$ for a typical test location on the semi-processed NOES sample (sample A).
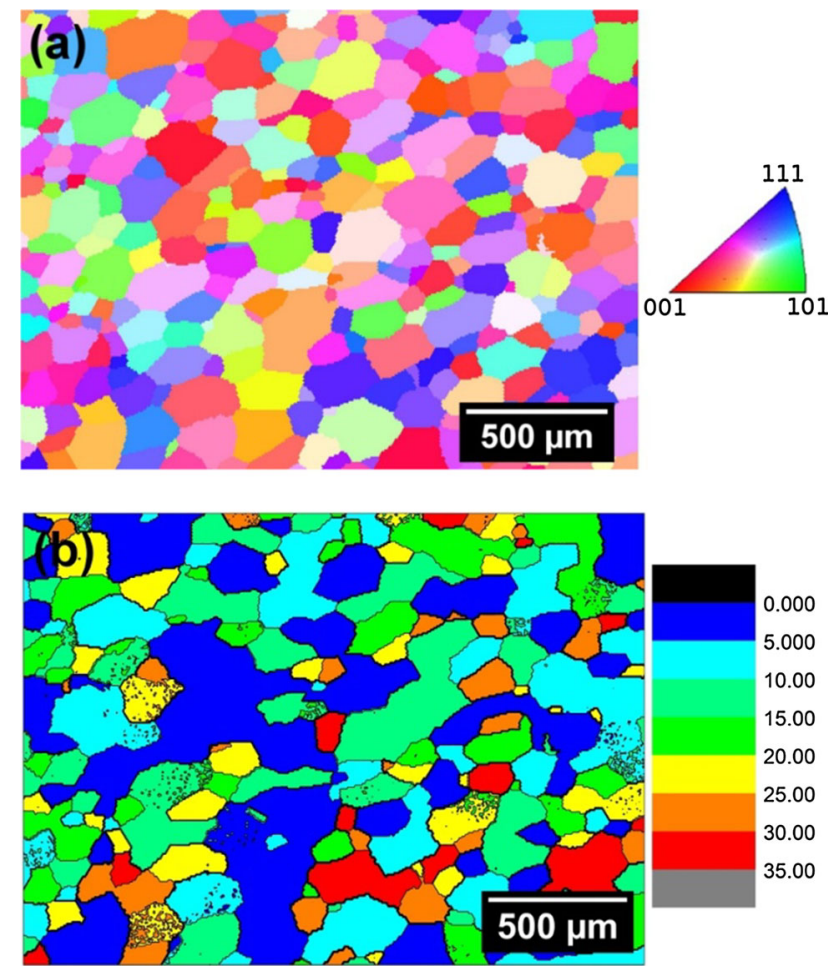

Fig. 5-Inverse pole figure (IPF) map in the ND $(a)$ and the $\beta$ parameter map $(b)$ for a typical test location on the fully processed NOES sample (sample B).

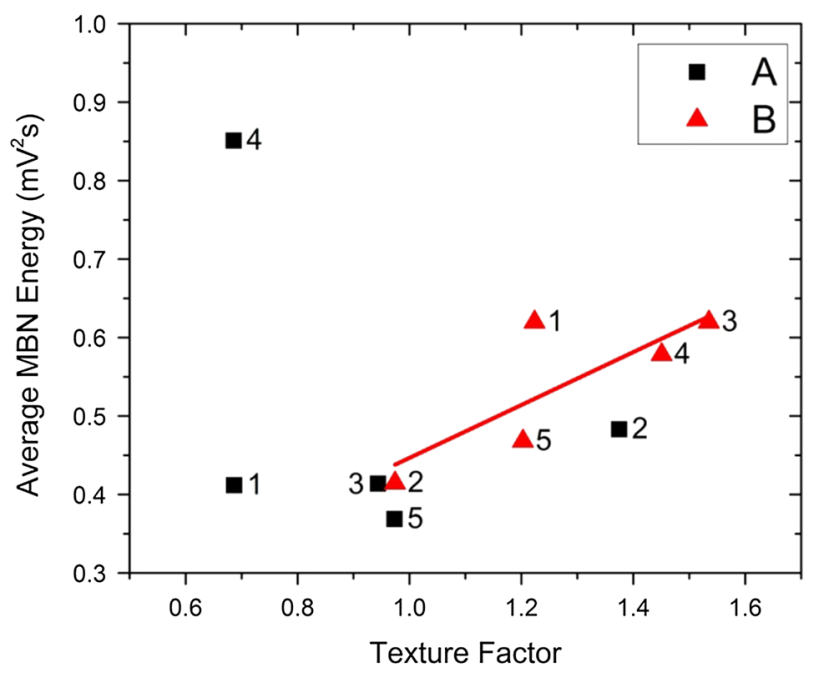

(a)

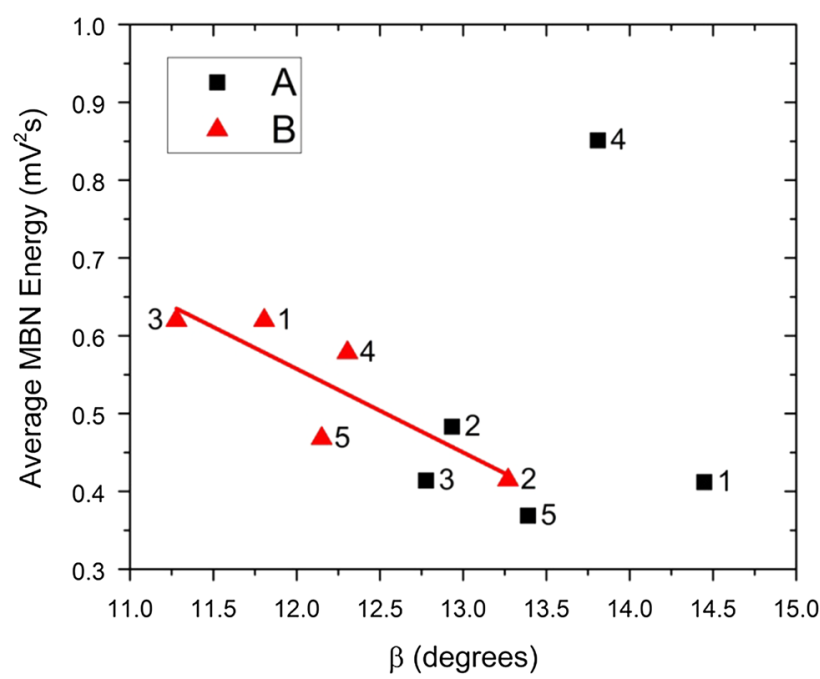

(b)

Fig. 6 - Graphs depicting MBN Energy averaged over 12 directions $v s$ texture factor $(a)$, and $\beta$ parameter $(b)$. In both cases, the semiprocessed sample (points A1-A5) did not exhibit enough of a trend to plot a trendline. For the fully processed samples (points B1-B5), the average MBN energy had a linear trend with both texture factor and $\beta$.

\section{Fully processed}

For the fully processed test locations, mild trends were observed between average MBN energy and TF (red triangles in Figure 6(a)) as well as $\beta$ parameter (red triangles in Figure 6(b)). In both cases, the trends matched established theory. A higher TF and a lower $\beta$ parameter indicated more easy axes aligned in the plane of interest for $\mathrm{MBN}$, and more easy axes present are associated with a higher MBN energy. ${ }^{[26]}$

The fit associated with the $\beta$ parameter appeared to be slightly better than TF for the fully processed sample. This was attributed to the fact that the calculation of $\beta$ involves every grain in the scan area, and accounts for the easy axis contribution from the $\{h k 0\}$ planes. However, the improvement in fit was quite small, and may not be statistically significant. The suspected 
reasons for this are twofold: (1) These samples had a relatively small amount of $\{h k 0\}$ planes present, approximately 10 pct within $15 \mathrm{deg}$ of $\{110\}$, and (2) the $\{100\}$ planes have two easy axis directions present (which is more beneficial for isotropic magnetic properties), and in most cases were a significant texture component. In other words, the $\beta$ parameter does not distinguish between $\{100\}$ (two easy axes present) and $\{110\}$ (one easy axis present), both of which would have a $\beta$ of 0 deg. As a result, the two texture parameters had a comparable accuracy.

\section{Magnetic Properties vs Misorientation}

In addition to the texture analysis, the relative orientation between neighboring grains was studied from the correlated MDF. A standard GBCD was calculated for each sample and testing location. A low angle grain boundary was defined as having a misorientation of between 2 and $15 \mathrm{deg}$, a large angle grain boundary of larger than $15 \mathrm{deg}$, and CSL boundaries $(\Sigma 3-\Sigma 27 b)$ as determined by the Brandon criterion. ${ }^{[27]}$ Similarly, the EAM parameter was calculated for each test location. This EAM parameter can be visualized as a measure of how closely the easy axes were aligned between neighboring grains.

\section{Semi-processed}

For the semi-processed sample locations, an approximate trend of increasing fraction of low angle GB's and decreasing average MBN energy was observed (black squares in Figure 7(a)). However, no clear trends of average MBN energy and the amount of CSL boundaries or high angle boundaries were observed (black

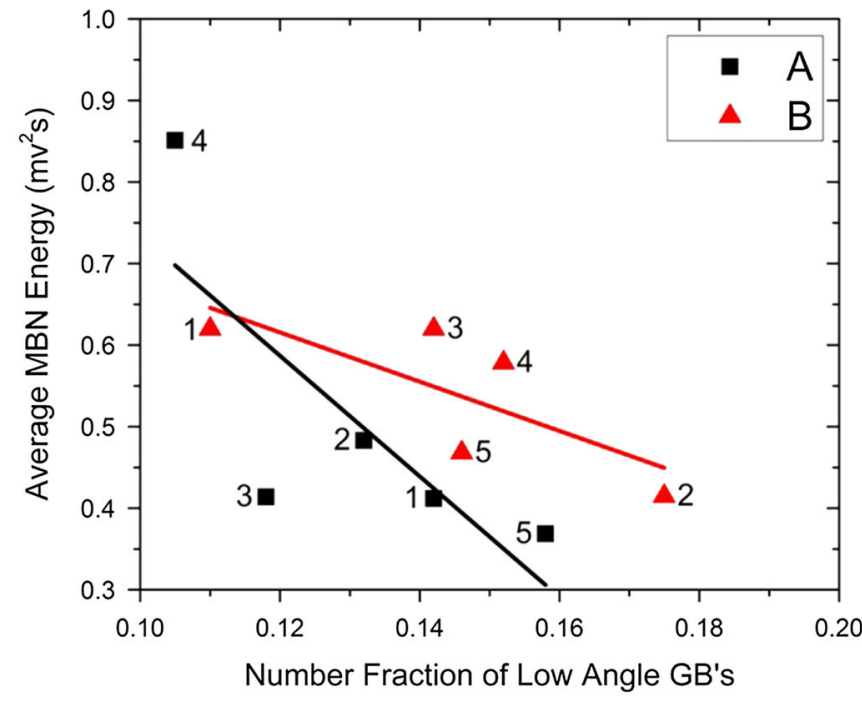

(a)

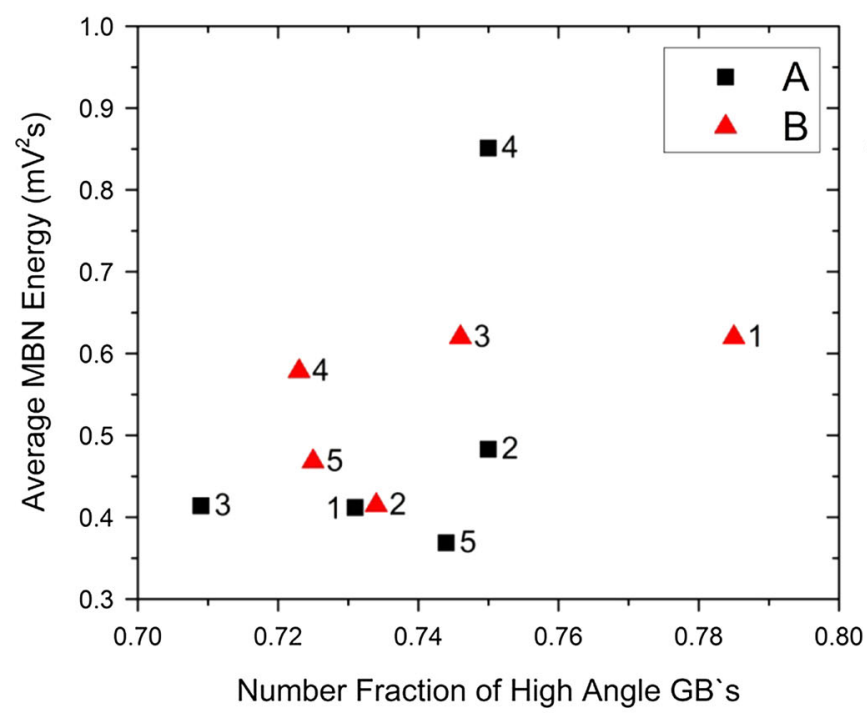

(c)

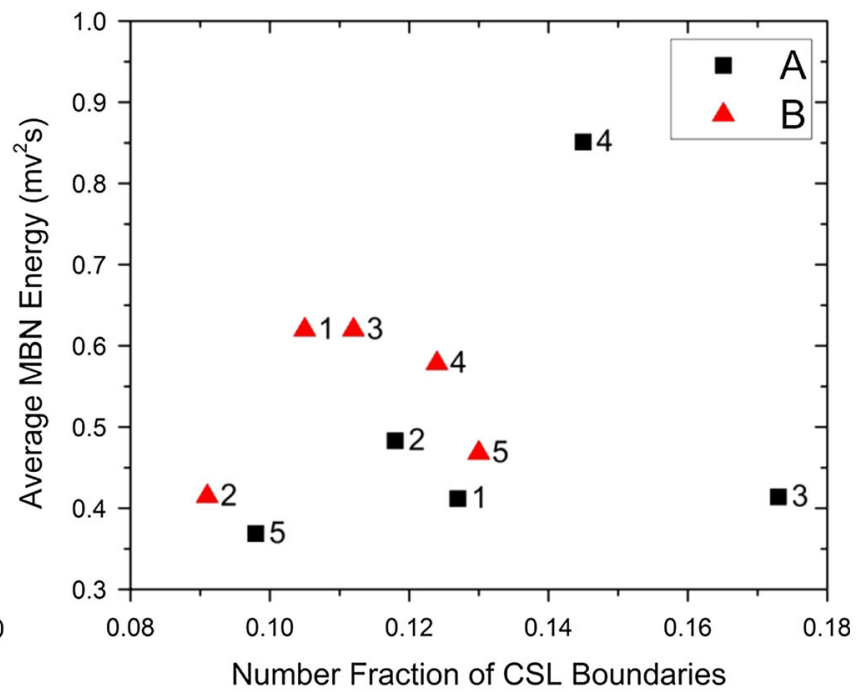

(b)

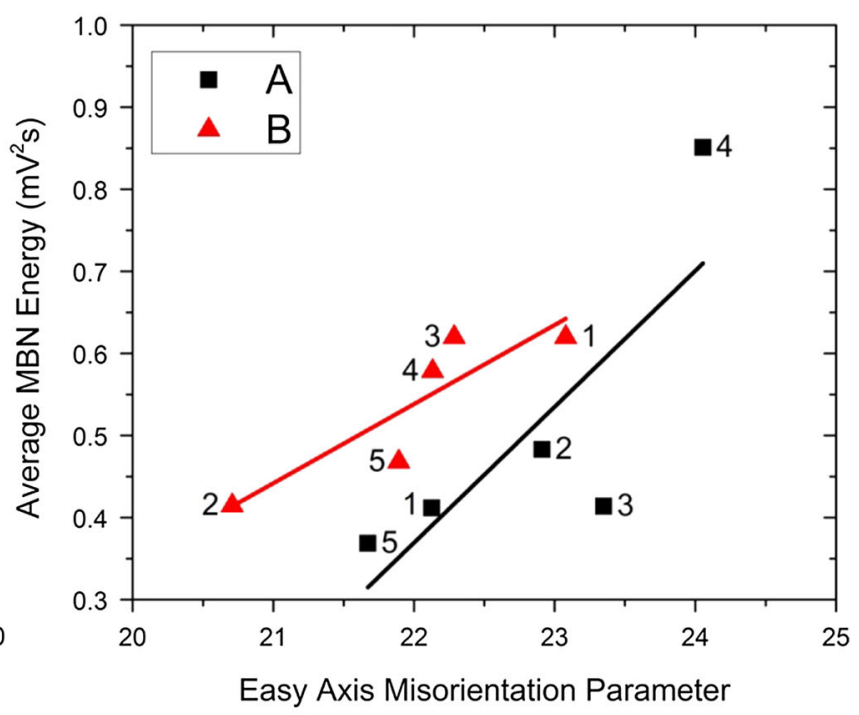

(d)

Fig. 7- Graphs showing the average MBN energy in 12 directions $v s$ percentage of low angle GB's (a), the percentage of CSL boundaries (b), and the percentage of high angle GB's $(c)$. Image $(d)$ shows the average MBN energy $v s$ the EAM parameter. 
squares in Figures 7(b) and (c)). This implies that the special class of CSL boundaries had little effect on the semi-processed NOES. If all of the misorientations greater than 15 deg were grouped together with no CSL consideration, then the opposite trend to Figure 7(a) would be present, since the number fraction of large angle boundaries would be 1 - (the number fraction of small angle boundaries).

When the EAM parameter was plotted with average MBN energy, a mild trend was observed (black squares in Figure 7(d)), where a larger EAM parameter resulted in a larger MBN energy. Although the linear fit did not appear to be significantly improved, the EAM parameter was able to explain the high average $\mathrm{MBN}$ energy for test location A4, which could not be explained by other previously examined texture parameters. This A4 sample had a larger EAM value than any other test location (semi-processed or fully processed) that was examined.

\section{Fully processed}

For traditional misorientation, the fully processed sample displayed the same relationship to average $\mathrm{MBN}$ energy as the semi-processed sample (see red triangles in Figures 7(a) through (c)). A mild trend was observed with respect to low angle GB's, and no influence of CSL boundaries was observed.

The trends between average MBN energy and EAM parameter (red triangles in Figure 7(d)) followed a fairly strong linear trend. This linear trend appeared to be more accurate than the other trends of texture parameters vs average $\mathrm{MBN}$ energy for the fully processed samples.

\section{Influence of misorientation}

From both interpretations of misorientation, a greater misorientation, or in the case of the EAM parameter, a greater misorientation of the easy axis, led to an increase in the average MBN energy in both the semi-processed and fully processed NOES samples. This matches with previous research conducted on GOES, where an increase in misorientation led to a higher MBN energy for low angle grain boundaries that were examined. ${ }^{[17]}$ Since a larger $\mathrm{MBN}$ energy has been associated with lower losses for NOES, ${ }^{[15]}$ an increase in EAM appears to be desirable in that it will decrease the losses. The trends associated with the EAM compared to the traditional misorientation were more prevalent for the fully processed sample. This was attributed to the fact that EAM is related to the misorientation of the easy axis, while traditional misorientation can involve any possible axis, and the two are not necessarily equivalent with respect to magnetic properties due to magnetocrystalline anisotropy energy.

\section{E. Easy Axis Misorientation Distributions}

In addition to examining average MBN energy vs the EAM parameter, the EAM distribution for each testing location was also represented as a $3 \mathrm{~d}$ scatter plot from the misorientation angle for each easy axis (Figure 8 through Figure 17).
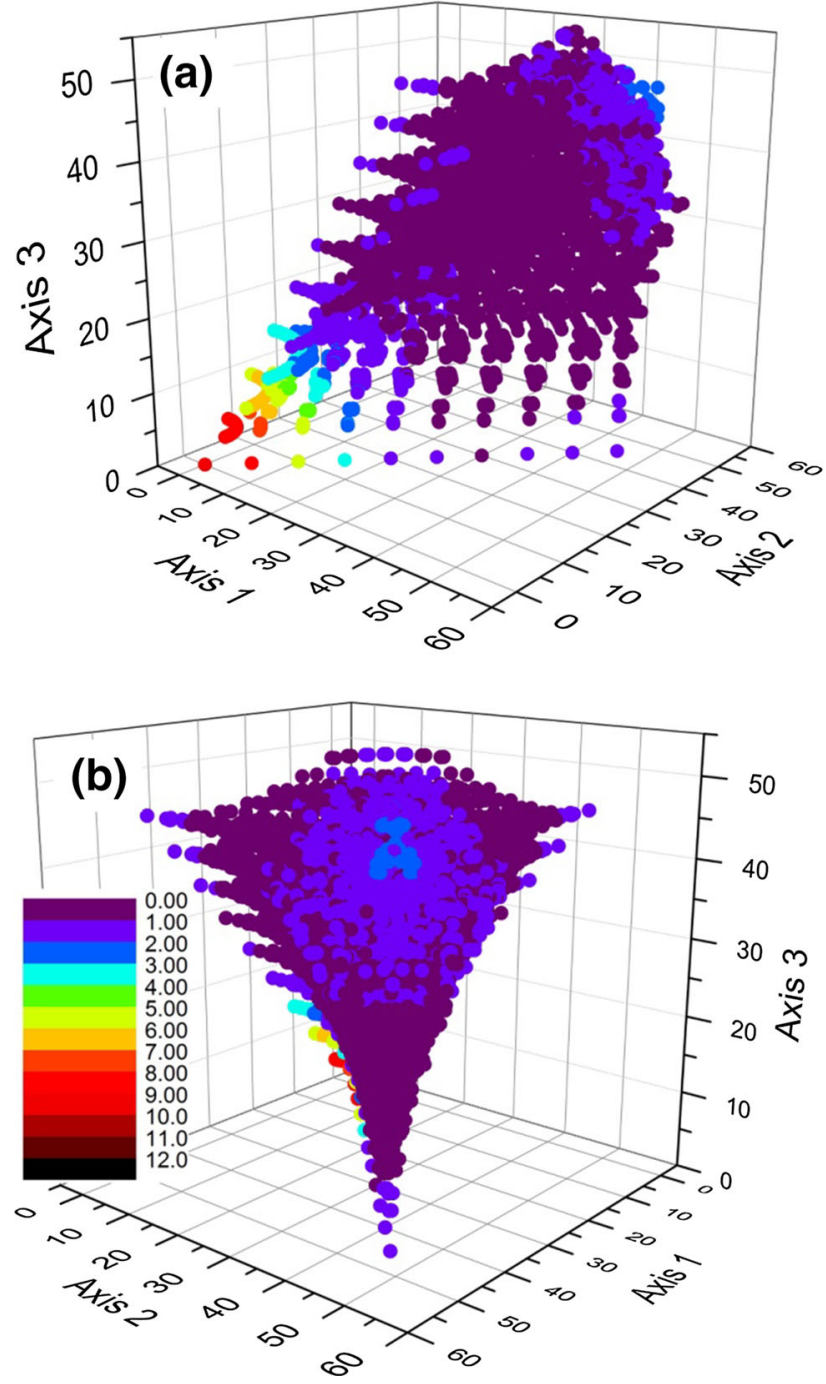

Fig. 8-Easy axis misorientation distribution plot for sample A1 showing two different views, one showing the distribution for the lower easy axis misorientations $(a)$, and one showing the distribution for the higher misorientations $(b)$.

\section{Semi-processed}

For the semi-processed sample (sample A), low angle EAMs close to the origin were similar for all test locations, except for A2, which showed a much lower intensity (Figure 9(a)), and A5, which had a slightly higher intensity (Figure 12(a)). Similarly, A1, A3, and A4 all showed slightly higher intensities for larger angle EAMs (Figures 8(b), 10(b), and 11(b), respectively), and A2 and A5 showed lower intensities for high angle misorientations, with A5 showing the lowest.

These distribution trends were reflected in the average MBN energy values. The A5 sample had the lowest average $\mathrm{MBN}$ energy value, and correspondingly showed the highest intensity of small EAM, and the lowest intensity of high misorientation. In other words, this A5 sample had the highest easy axis alignment between neighboring grains, and also the lowest average MBN energy. 

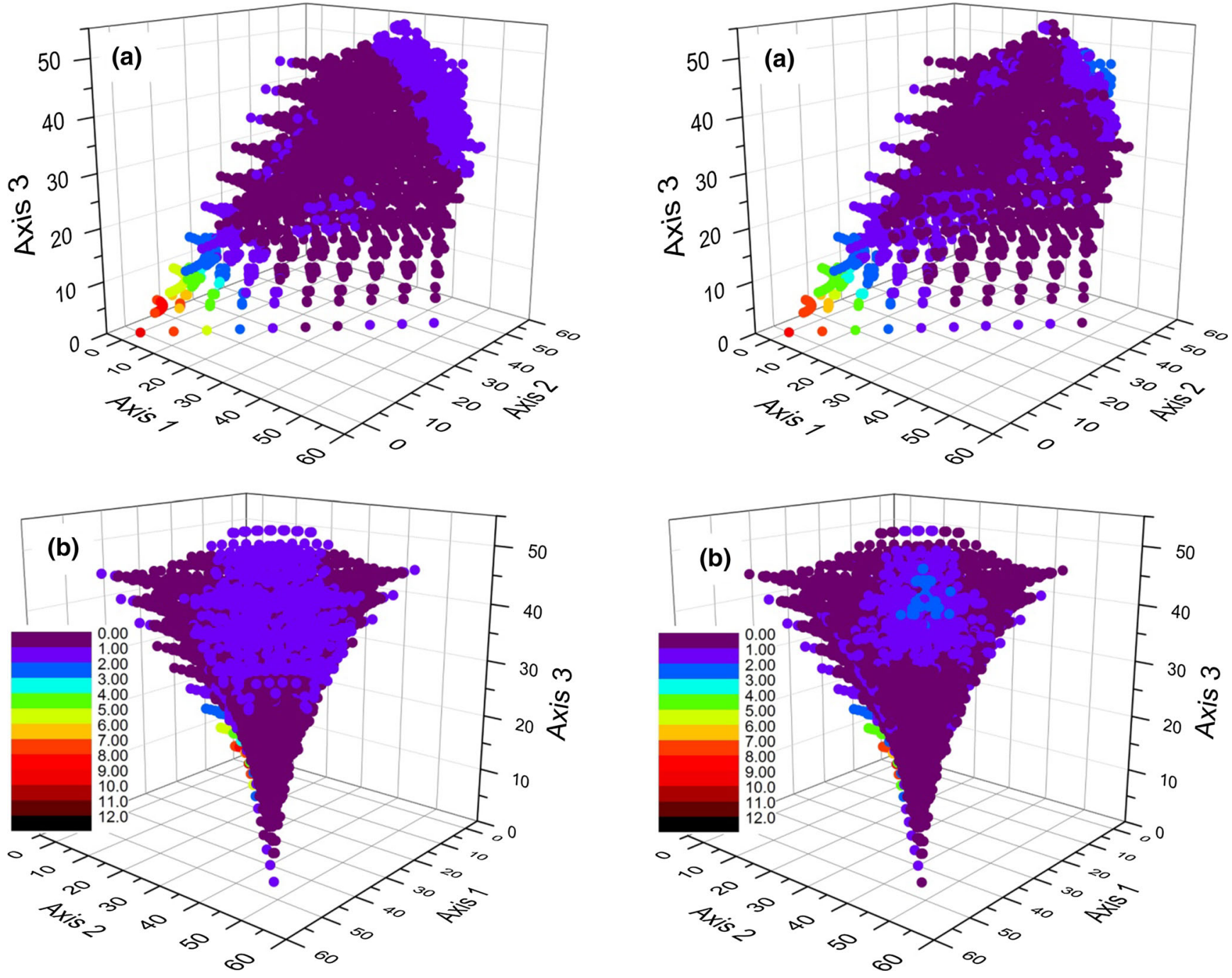

Fig. 9-Easy axis misorientation distribution plot for sample A2 showing two different views, one showing the distribution for the lower easy axis misorientations (a), and one showing the distribution for the higher misorientations $(b)$.

Similarly, the A4 sample showed significantly less small angle EAM than any of the other samples examined, along with a larger amount of high angle misorientation intensity. Consequently, this sample had less easy axis alignment between neighboring grains, and showed the largest average MBN energy by a significant margin.

\section{Fully processed}

For the fully processed samples, similar trends were observed. For the low angles of EAM, especially for zero, sample B2 had the highest intensity/alignment, and also the lowest average MBN energy value. B5 had the second highest intensity for the low angle misorientation, and possessed the second lowest average MBN energy. Samples B3 and B4 had the highest intensity for the large angles of EAM, and had higher values for average MBN. These results are a further description of the fact that a higher EAM parameter, or a greater EAM, led to a higher MBN energy.

Fig. 10-Easy axis misorientation distribution plot for sample A3 showing two different views, one showing the distribution for the lower easy axis misorientations $(a)$, and one showing the distribution for the higher misorientations $(b)$.

\section{DISCUSSION}

\section{A. Semi-processed Vs Fully Processed Isotropic Magnetic Properties}

For the semi-processed (A) and fully processed (B) samples that were examined, the difference was that the fully processed samples had been subjected to an additional stress relief annealing process prior to testing. As a result, the following differences in the metallurgical parameters were observed:

- The fully processed samples had higher TF values, with a smaller spread in values, indicating a more consistent and favorable local texture when compared to the semi-processed sample. Similarly, the fully processed samples displayed lower values of $\beta$ parameter.

- The magnetic properties of the fully processed samples were more accurately predicted as linear trends by both the TF, $\beta$, and also the EAM parameter. 

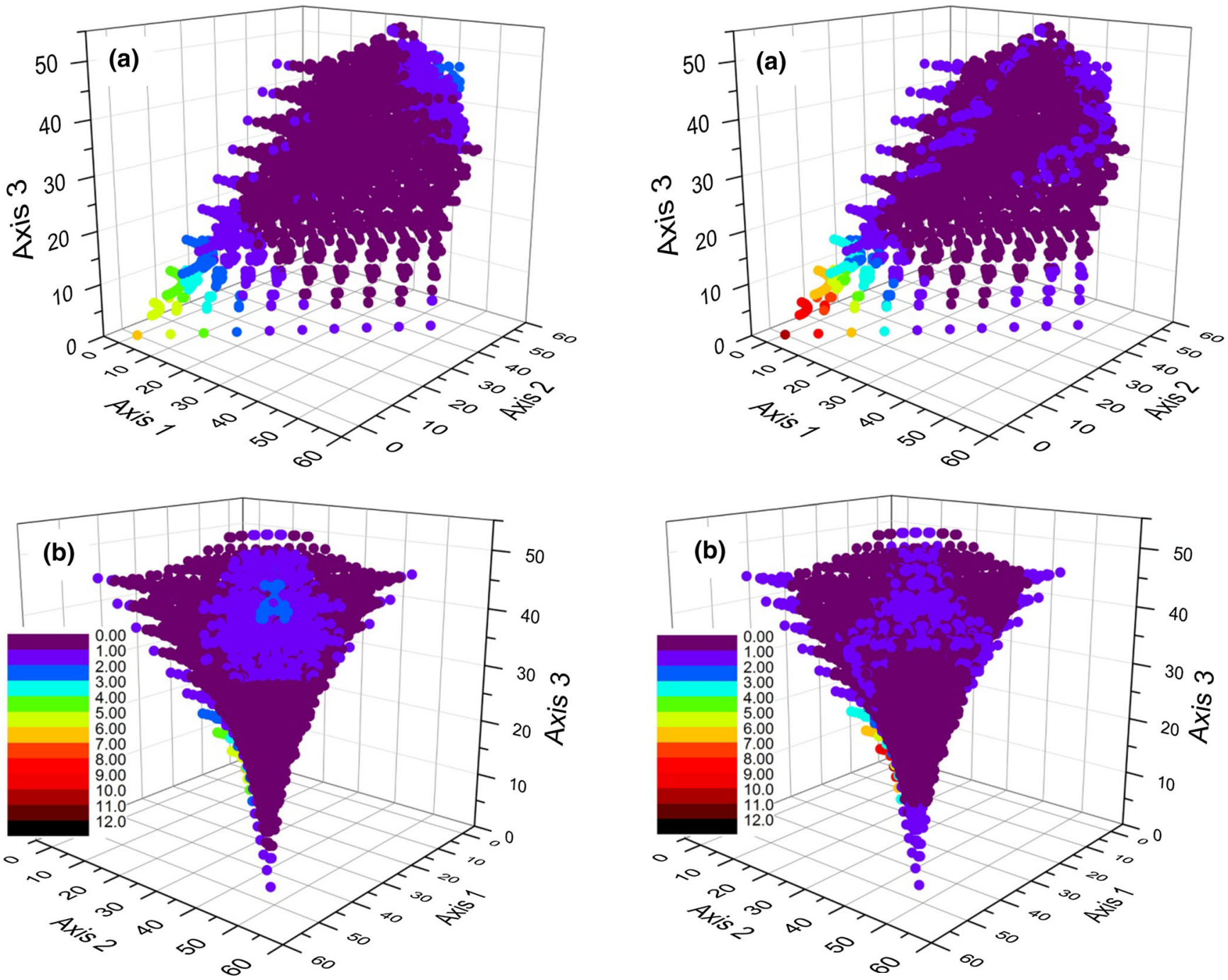

Fig. 11-Easy axis misorientation distribution plot for sample A4 showing two different views, one showing the distribution for the lower easy axis misorientations (a), and one showing the distribution for the higher misorientations $(b)$.

- The fully processed samples showed a comparable intensity for the high angle values of EAM, but a higher intensity for the low angle values of EAM when compared to the semi-processed samples.

When comparing the average MBN energy values, in most cases, the fully processed samples had a slightly higher MBN energy than the semi-processed samples. This was attributed to the magnetic property improvement from texture indicated by a larger $\mathrm{TF}$ and a smaller $\beta$, describing more easy axes lying in the RDTD plane of the sheet.

The decreased accuracy in the prediction of the magnetic properties of the semi-processed samples (A) was attributed to a few factors. First, the larger local variations in texture would lead to the larger variation in MBN energy at the various testing locations. The test point that did not match well with the others in prediction of magnetic properties (A4) was found to

Fig. 12-Easy axis misorientation distribution plot for sample A5 showing two different views, one showing the distribution for the lower easy axis misorientations $(a)$, and one showing the distribution for the higher misorientations $(b)$.

have a much lower intensity than any of the other samples for lower angle EAM in the distribution graphs. This was the likely reason for this sample's much higher average MBN value (discussed in more detail in the following sections), which made many of the trend lines with respect to orientation difficult to fit for the semiprocessed samples.

In addition to these factors, variations in residual stress in the semi-processed samples may also have been a factor in the magnetic properties, since these samples were not subjected to the additional stress relief annealing process. The presence of residual stress, especially due to cold deformation from manufacturing processes, will shift the maximum MBN energy location to the closest $\langle 100\rangle$ direction to the tensile stress direction. ${ }^{[28,29]}$ As a result, the MBN energy may no longer trend with calculated texture parameters, since the directional (and therefore average) MBN energy values would be partially based on the magnitude and direction of residual stress. 

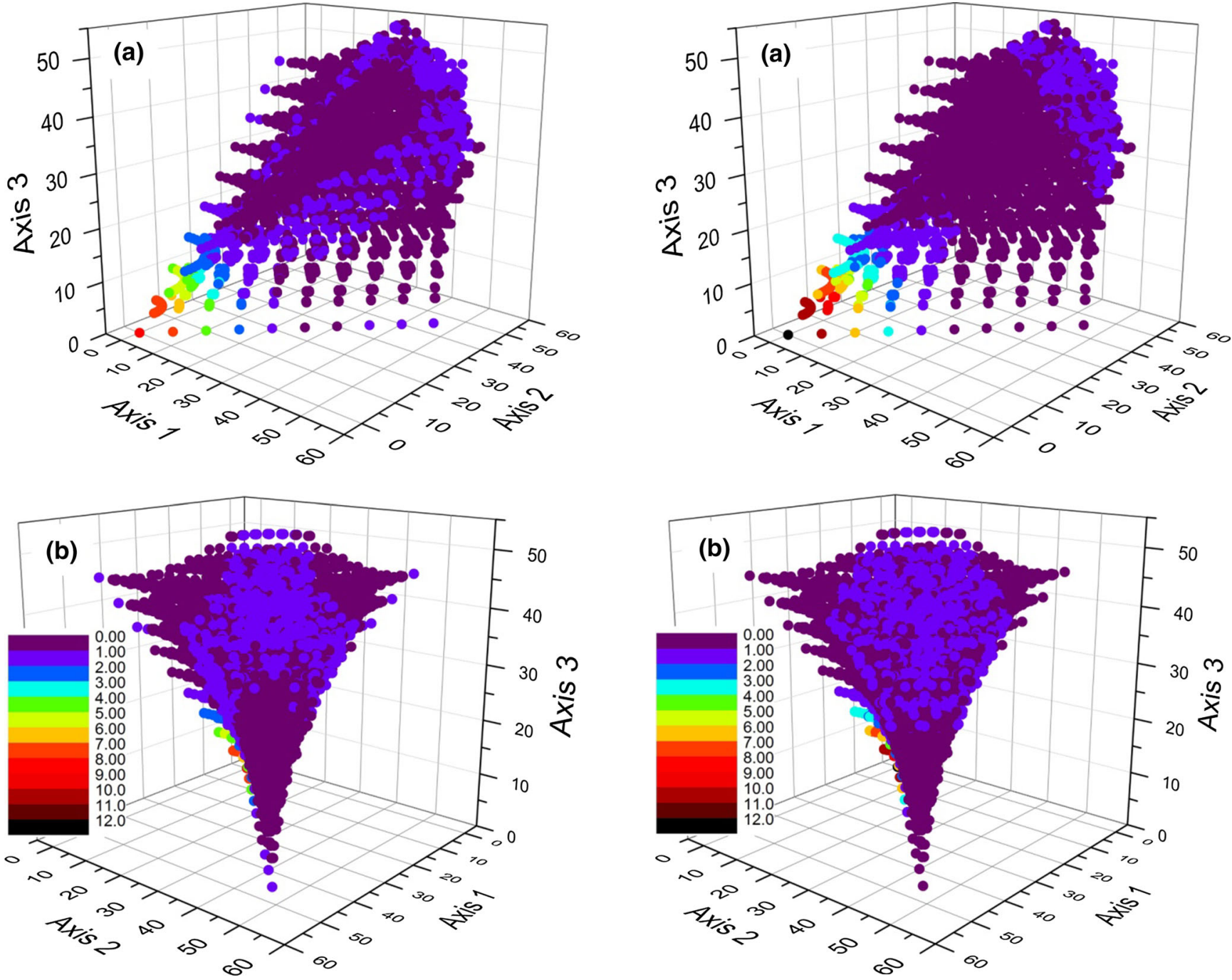

Fig. 13-Easy axis misorientation distribution plot for sample B1 showing two different views, one showing the distribution for the lower easy axis misorientations $(a)$, and one showing the distribution for the higher misorientations $(b)$.

Although residual stress was likely a factor, when the EAM was considered, the magnetic property prediction for the semi-processed samples was improved to account for point A4. The additional reduced accuracy in the fit for the semi-processed samples in the EAM graph may be a result of local variations in residual stress, however, there are also other possible reasons for additional data scatter discussed in Section IV-C.

\section{B. Overall Interpretation of EAM}

From both sets of samples, it appeared that the intensity of EAM for low angles was more influential on the magnetic properties compared to high angles of EAM. In other words, a low amount of low angle EAM intensity increased the average MBN energy to a greater extent than a high intensity of high angle EAM. The reason for this is believed to be domain continuity across grain boundaries. When the easy axes align well across the grain boundary (with the same angle with

Fig. 14-Easy axis misorientation distribution plot for sample B2 showing two different views, one showing the distribution for the lower easy axis misorientations $(a)$, and one showing the distribution for the higher misorientations $(b)$.

respect to the GB normal), there is a minimum of magnetic free poles, and as a result the domain structure can be continuous, or continuous with closure domains. ${ }^{[18,20,30]}$ It was recently hypothesized that this might be beneficial to the magnetic properties of NOES based on a reduction of magnetostatic energy, minimizing the losses associated with complex domain structures and also from the pinning effects of grain boundaries. ${ }^{[20]}$ This hypothesis was also suggested by work of Kawahara et al., which revealed less interaction between the domain walls and a low angle GB than a high angle boundary under an applied field. ${ }^{[18]}$ However, from the results discussed, this domain continuity in NOES associated with low EAM values appears to decrease the MBN energy, which would be consistent with an increase in total loss. ${ }^{[15,21]}$

If the magnetic domain structure is aligned across the grain boundary, from a magnetic point of view it is essentially increasing the effective grain diameter of the material. Moreover, domain continuity was recently 

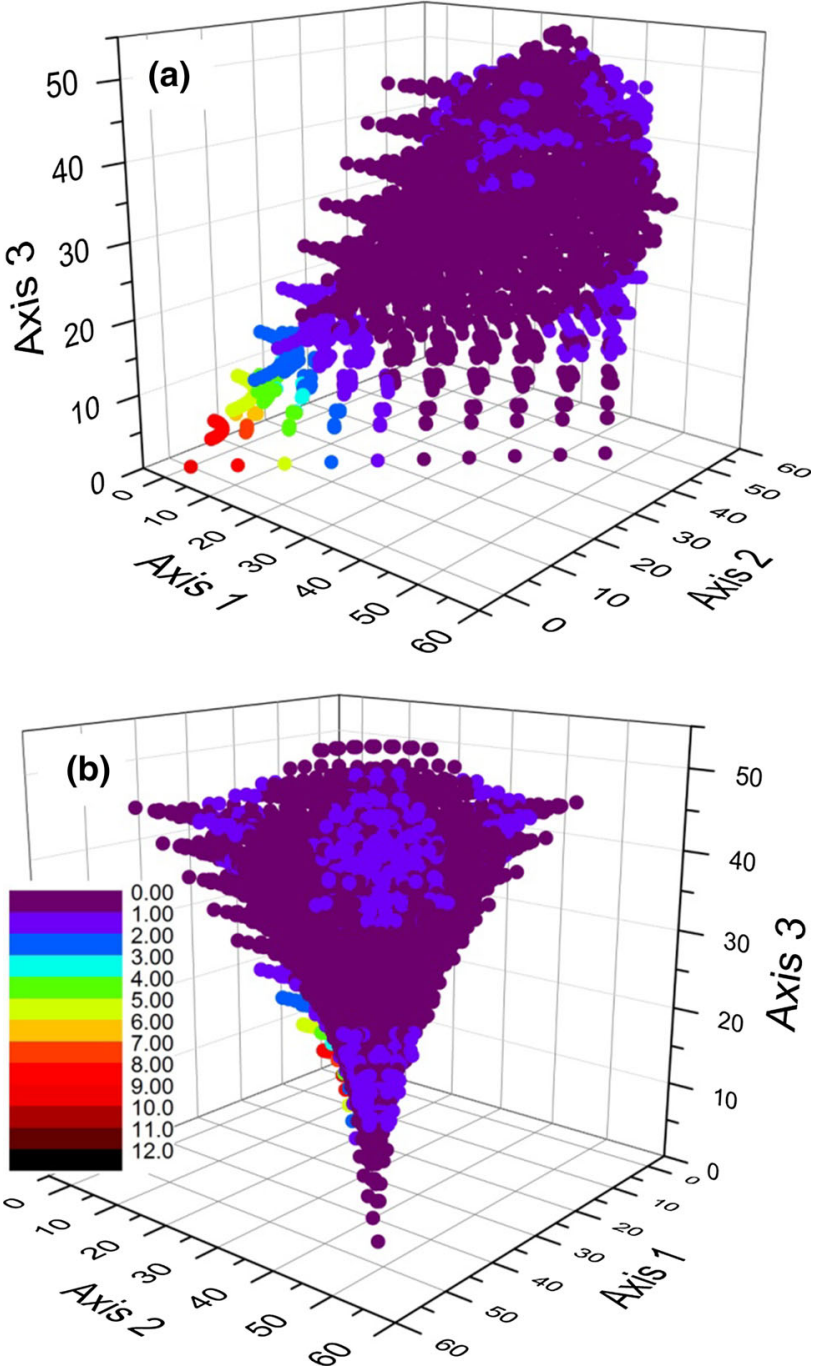

Fig. 15-Easy axis misorientation distribution plot for sample B3 showing two different views, one showing the distribution for the lower easy axis misorientations (a), and one showing the distribution for the higher misorientations $(b)$

observed to increase the width of the domain structure in NOES for a given misorientation of the easy axis and the RD-TD plane. ${ }^{[20]}$ Given the grain diameter range of these samples $(\sim 135$ to $150 \mu \mathrm{m})$, an increase in grain diameter will increase the total loss, since an approximate guideline value for minimum loss occurs at approximately $150 \mu \mathrm{m} .^{[2,31]}$

The effect of grain size on losses is divided between influences from hysteresis losses (where GB's are acting as sites for domain wall formation and annihilation) and excess/anomalous losses (eddy current effects associated with domain walls). ${ }^{[32]}$ Hysteresis loss increases with the inverse of grain diameter, which approximates the grain boundary area per unit volume. ${ }^{[32]}$ Excess losses increase in proportion to the domain width, and from this, excess losses are proportional to the square root of grain diameter. ${ }^{[31,32]}$ The total loss for these samples is related to the minimum of these two loss components (with bulk scale classical loss being constant since the thickness and resistivity of the samples tested were constant).
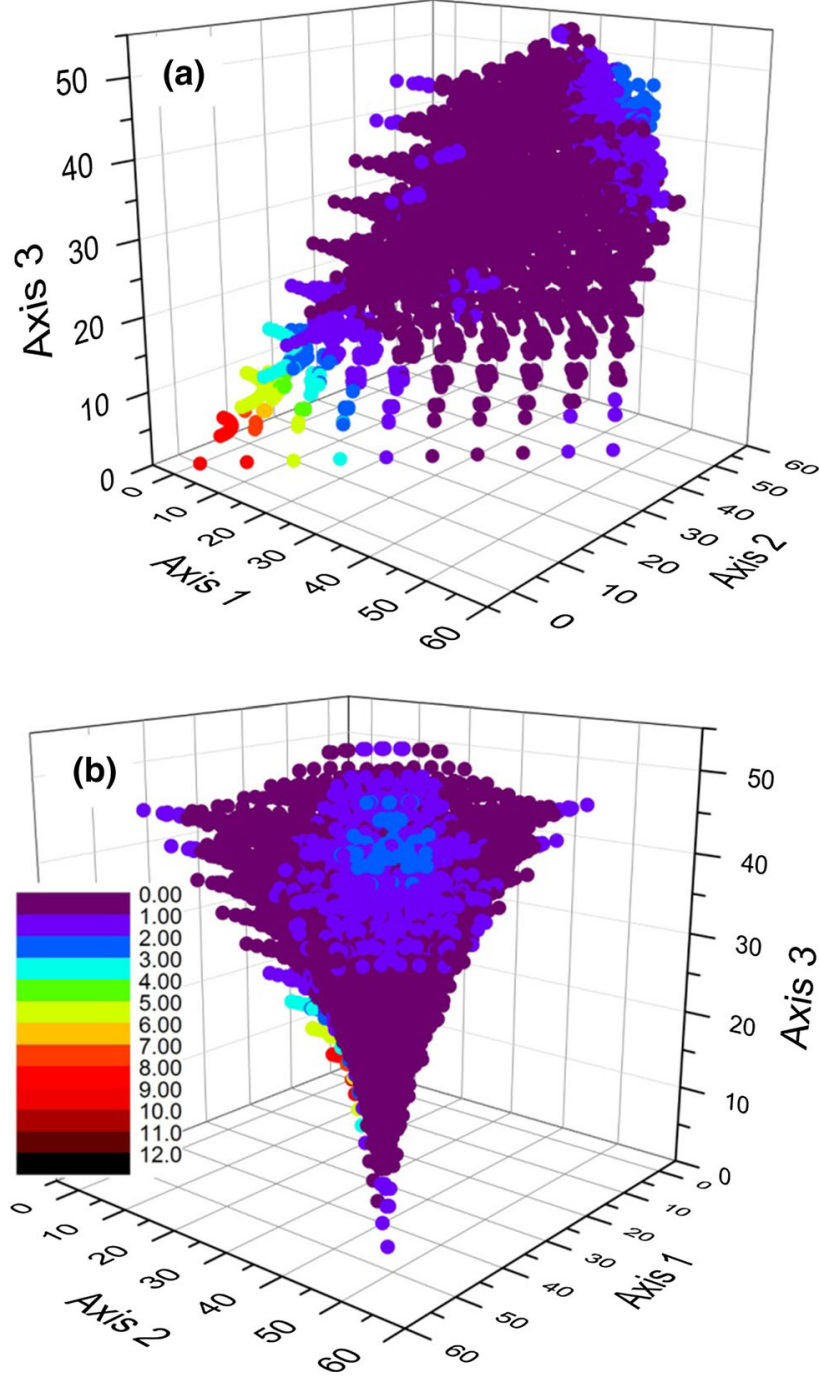

Fig. 16-Easy axis misorientation distribution plot for sample B4 showing two different views, one showing the distribution for the lower easy axis misorientations $(a)$, and one showing the distribution for the higher misorientations $(b)$.

When the effective grain size and domain width increase due to domain continuity from easy axis alignment, the excess losses will increase. For effective grain diameter values above the minimum loss value, the total loss will be due to the increase in excess loss. ${ }^{[32]}$

This explains the trends of EAM parameter and EAM distribution $v s$ average MBN energy. The intensity of the low angles of EAM is related to the continuity of the domains across the grain boundary. If this intensity is high (large amounts of continuity), the effective grain size will be larger, and excess losses (and therefore total losses) increase, as shown by a decrease in MBN energy. As the angle of the EAM increases above the low angles associated with domain continuity, further increases do not affect continuity, meaning that there is no effective change in the grain diameter. As a result, changes in EAM at larger angles would not be expected to significantly affect excess losses, but further EAM increases may reduce losses by enhancing the nucleation of reverse magnetic domains. This is suggested by the 

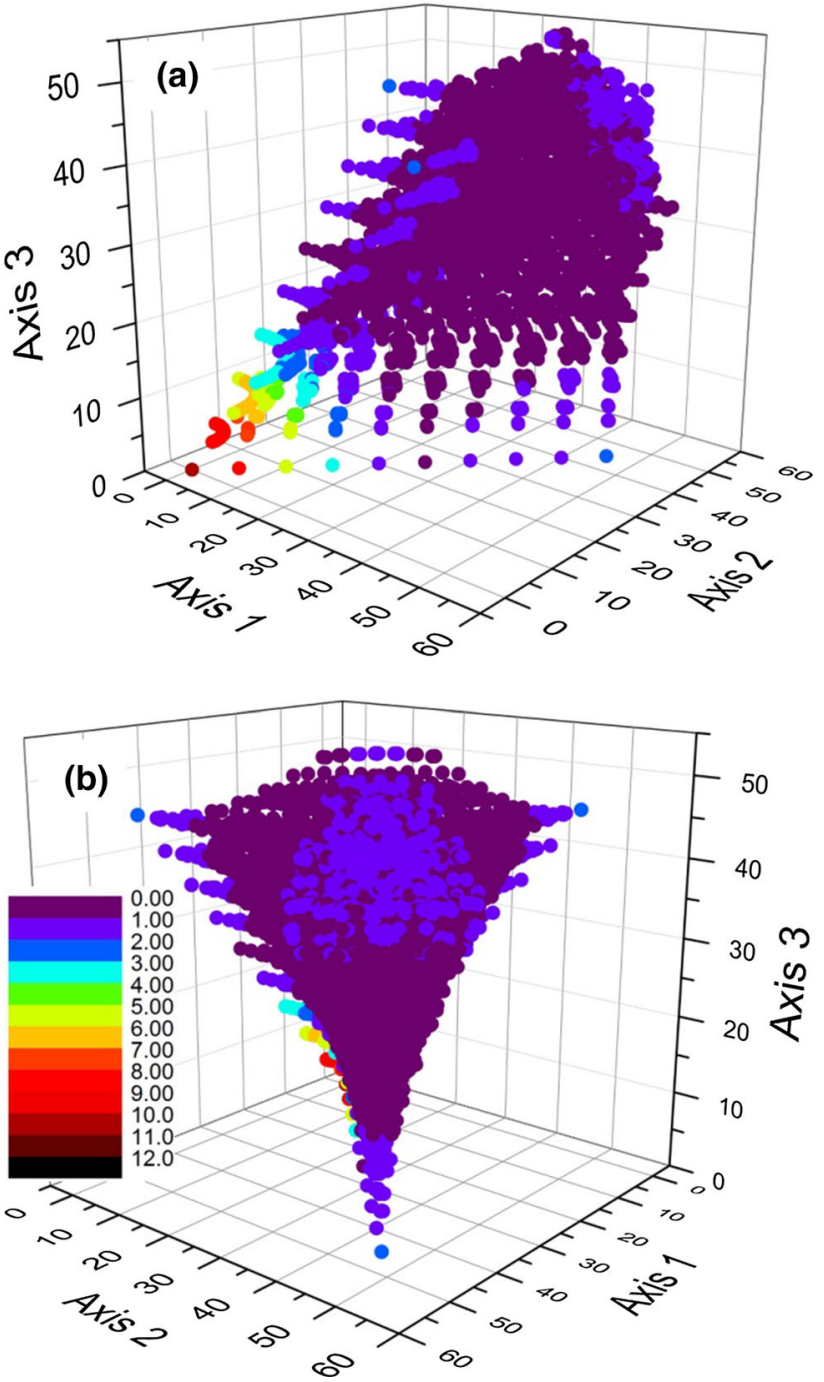

Fig. 17-Easy axis misorientation distribution plot for sample B5 showing two different views, one showing the distribution for the lower easy axis misorientations (a), and one showing the distribution for the higher misorientations $(b)$.

work of Goodenough who demonstrated that an increased amount of magnetic free poles across a grain boundary produced a larger the driving force for reverse domain nucleation. ${ }^{[33]}$ The results presented suggest that the effect reducing the domain continuity at lower angles of EAM is more beneficial than the enhanced domain nucleation at high EAM values.

\section{Final Summary of Orientation/Misorientation $V S$ Easy Axis Interpretations}

The results discussed above have shown the importance in considering both orientation and misorientation with respect to the magnetic easy axis directions as opposed to the traditional definitions. Previously, misorientation-related parameters had not been considered for NOES, since standard misorientation and GBCD values are not directly related to the magnetic easy axis.
Although originally intended as a supplemental texture parameter that could be used to explain deviant data points in magnetic property/texture property graphs, EAM has shown to be fairly accurate in predicting magnetic properties when compared to traditional texture properties. However, this accuracy at predicting magnetic properties independently may have been aided by the fact that for both the semi-processed and fully processed samples, all of the test locations were from the same strip. With grain size, chemistry, and thickness all constant, the influence of other variables influencing hysteresis and classic loss terms would be minimized, such that the effect of EAM on the excess loss would be more apparent.

In terms of the EAM trend lines, two factors that may have contributed to the moderate fit accuracy were as follows: (1) The EAM parameter was calculated by averaging the three angles and applying the normalized MDF weight. This is a simple way to obtain an overall idea of the EAM, but with results suggesting that the intensity of smaller angle EAMs were more influential than the higher angle EAMs, this may have influenced the fit quality. As a result, it is important to look at the EAM distribution as well as the EAM parameter value in order to capture the overall picture. (2) Although the misorientation of the easy axis is being calculated in the EAM, this calculation does not include the geometry of the grain boundary normal, which is used to calculate the magnetic free pole density (influencing the likelihood of domain continuity). It has been shown that the orientation of the GB does affect the domain structure and the material's properties. ${ }^{[30]}$ This may result in some inaccuracies, since EAM is a measure of EAM between two crystal orientations, rather than the misorientation between the easy axes from the two crystals and the grain boundary normal. However, the EAM parameter and distribution provide reasonable predictive capabilities compared to existing texture parameters, and are relatively easy to calculate.

\section{CONCLUSIONS}

A way to non-destructively measure isotropic magnetic properties in NOES was presented as an attempt to reveal properties that would be more representative of the steel's behavior under rotating magnetic fields. As a result, the following conclusions were made:

1. Significant local scale changes in magnetic properties, orientation, and misorientation measurements were observed for different locations on strips of semi-processed and fully processed NOES.

2. The magnetic properties at a local scale were more difficult to predict for the semi-processed sample using orientation and misorientation parameters when compared to the fully processed sample. This was attributed to more variation in local texture, more variation in low angle EAM values, as well as the possible influence of residual stress.

3. $\beta$ parameter was shown to be comparable to traditional texture factor at predicting the isotropic mag- 
netic properties provided by average $\mathrm{MBN}$ energy.

4. EAM calculations were shown to be more effective than traditional misorientation values at predicting the magnetic properties with a constant chemistry, grain size, and thickness. The EAM parameter was also shown to be slightly more accurate at predicting the magnetic properties than either $\beta$ or TF.

5. A larger EAM parameter value, i.e., less easy axis alignment between grains, was associated with a higher MBN energy value (and therefore lower losses). This was chiefly attributed to a decrease in the excess loss related to domain alignment across grain boundaries represented by the low angles of EAM.

6. Although a high average EAM parameter is desired to maximize MBN energy (minimizing total loss), a low intensity for low EAM angles (attributed to less domain continuity) appeared to be more beneficial to the magnetic properties than a corresponding high intensity for a high angle EAM. This may imply that the detrimental effect of domain continuity at low EAM is more significant overall than the beneficial effect of enhanced reverse domain nucleation at high EAM in determining the magnetic properties.

\section{ACKNOWLEDGMENTS}

This work was supported by the Natural Science and Engineering Research Council (NSERC) of Canada, Collaborative Research and Development Grants Program. MG would like to thank Ms. Yaoyao Ding and Dr. Pampa Ghosh for their helpful discussions in the writing of this paper. The authors gratefully acknowledge the donation of the NOES samples from General Motors Canada.

\section{OPEN ACCESS}

This article is distributed under the terms of the Creative Commons Attribution License which permits any use, distribution, and reproduction in any medium, provided the original author(s) and the source are credited.

\section{REFERENCES}

1. K. Honma, T. Nozawa, H. Kobayashi, Y. Shimoyama, I. Tachino, and K. Miyoshi: IEEE Trans. Magn., 1985, vol. MAG-21(5), pp. 1903-08.
2. K. Matsumura and B. Fukuda: IEEE Trans. Magn., 1984, vol. 20 (5), pp. 1533-38

3. S. Chikazumi: Physics of Ferromagnetism, Oxford University Press Inc., New York, 2010, pp. 249-56.

4. R. PremKumar, I. Samajdar, N.N. Viswanathan, V. Singal, and V. Seshadri: J. Magn. Magn. Mater., 2003, vol. 264 (1), pp. 75-85.

5. A. Chaudhury, R. Khatirkar, N.N. Viswanathan, V. Singal, A. Ingle, S. Joshi, and I. Samajdar: J. Magn. Magn. Mater., 2007, vol. 313 (1), pp. 21-28.

6. P. Ghosh, R.R. Chromik, B. Vashegi, and A.M. Knight: J. Magn. Magn. Mater., 2014, vol. 365, pp. 14-22.

7. L. Kestens and S. Jacobs: Texture Stress Microstruct., 2008, vol. 2008, Article ID 173083.

8. J. Barros, J. Schneider, K. Verbeken, and Y. Houbaert: J. Magn. Magn. Mater., 2008, vol. 320 (20), pp. 2490-93.

9. Y. Houbaert, T. Ros-Yáñez, A. Monsalve, and J. Barros Lorenzo: Phys. B, 2006, vol. 384 (1-2), pp. 310-12.

10. J. Barros, A. Targhetta, O. León, T. Ros, J. Schneider, and Y. Houbaert: J. Magn. Magn. Mater., 2007, vol. 316(2 Spec. Iss.), pp. e865-67.

11. E. Gomes, J. Schneider, K. Verbeken, J. Barros, and Y. Houbaert: IEEE Trans. Magn., 2010, vol. 46 (2), pp. 310-13.

12. J.J. Sidor, K. Verbeken, E. Gomes, J. Schneider, P.R. Calvillo, and L.A.I. Kestens: Mater. Charact., 2012, vol. 71, pp. 49-57.

13. T. Yonamine and F.J.G. Landgraf: J. Magn. Magn. Mater., 2004, vols. 272-276 (Suppl. 1), pp. e565-66.

14. H.G. Kang, K.M. Lee, M.Y. Huh, J.S. Kim, J.T. Park, and O. Engler: J. Magn. Magn. Mater., 2011, vol. 323 (17), pp. 2248-53.

15. A. Samimi, T. Krause, L. Clapham, M. Gallaugher, Y. Ding, P. Ghosh, R. Chromik, and A. Knight: J. Nondestruct. Eval., 2014, vol. 33 (4), pp. 663-69.

16. K.M. Lee, S.Y. Park, M.Y. Huh, J.S. Kim, and O. Engler: J. Magn. Magn. Mater., 2014, vol. 354, pp. 324-32.

17. S. Yamaura, Y. Furuya, and T. Watanabe: Acta Mater., 2001, vol. 49 (15), pp. 3019-27.

18. K. Kawahara, Y. Yagyu, S. Tsurekawa, and T. Watanabe: Mater. Res. Soc. Symp. P., 2000, vol. 586, pp. 169-74.

19. S. Chikazumi: Physics of Ferromagnetism, Oxford University Press Inc., New York, 2010, pp. 457-63.

20. M. Gallaugher, N. Brodusch, R. Gauvin, and R.R. Chromik: Ultramicroscopy, 2014, vol. 142, pp. 40-49.

21. M. Birsan, J.A. Szpunar, T.W. Krause, and D.L. Atherton: $J$. Appl. Phys., 1996, vol. 79(8 Part 2B), pp. 6042-44.

22. O. Engler and V. Randle: Introduction to Texture Analysis, 2nd ed., CRC Press, Taylor \& Francis Group, Boca Raton, FL, 2010, pp. $15-50$

23. S. White, T.W. Krause, and L. Clapham: IEEE Trans. Instrum. Meas., 2012, vol. 61 (7), pp. 1896-1907.

24. S. White: PhD Thesis, Queen's University, 2009.

25. S. White, T. Krause, and L. Clapham: Meas. Sci. Technol., 2007, vol. 18 (11), pp. 3501-10.

26. T.W. Krause, L. Clapham, and D.L. Atherton: J. Appl. Phys., 1994, vol. 75 (12), pp. 7983-88.

27. G. Brandon: Acta Metall., 1966, vol. 14 (11), pp. 1479-84.

28. L. Clapham, C. Heald, T. Krause, D.L. Atherton, and P. Clark: $J$. Appl. Phys., 1999, vol. 86 (3), pp. 1574-80.

29. L. Clapham, S. White, J. Lee, and D.L. Atherton: J. Appl. Phys., 2000, vol. 88 (4), pp. 2163-65.

30. S. Shin, R. Schafer, and B.C. De Cooman: IEEE Trans. Magn., 2010, vol. 46 (9), pp. 3574-81.

31. M. Shiozaki and Y. Kurosaki: J. Mater. Eng., 1989, vol. 11 (1), pp. $37-43$.

32. M.F. De Campos, J.C. Teixeira, and F.J.G. Landgraf: J. Magn. Magn. Mater., 2006, vol. 301 (1), pp. 94-99.

33. J.B. Goodenough: Phys. Rev., 1954, vol. 95 (4), pp. 917-32. 